\title{
Brinkmann, Janis: Journalismus. Eine praktische Einführung.
}

\author{
Baden-Baden: Nomos 2021. 276 Seiten. Preis: $€ 25$
}

\author{
Marlis Prinzing
}

Angenommen: 22. Dezember 2021 / Online publiziert: 11. Januar 2022

(C) Der/die Autor(en) 2022

Journalismus ist ein Beruf mit hoher Verantwortung und zudem unverzichtbar für unsere demokratische, digitale Mediengesellschaft. Journalismus organisiert kontinuierlich die Debatte über aktuelle Herausforderungen, die uns alle angehen. Er ist eine bedeutsame Instanz, die warnt, handlungsfähig macht, unterhält und uns $\mathrm{Zu}$ gänge zu den Informationen liefert, die wir benötigen, um uns eine eigene Meinung zu bilden und um kundig wählen zu können. Er hat die Aufgabe, Mächtige aller Gesellschaftsbereiche kritisch zu beobachten und zu kontrollieren und er bildet als „,vierte Macht“ das Rückgrat der Demokratie, deren Bestandsträger er ist. Auf diesen Säulen baut zumindest das Gros der Ausbildungsangebote im Journalismus in Deutschland auf - und auf der kontinuierlichen Reflexion darüber, unter welchen Bedingungen diese Leistungen erbracht werden beziehungsweise wann stattdessen Fehlleistungen stattfinden und worin diese bestehen.

Nun gibt es ein weiteres Lehrbuch „Journalismus“. Autor ist Janis Brinkmann. Er ist Professor für Publizistik in der digitalen Informationswirtschaft an der Hochschule Mittweida. In seinem Lehrprofil zählt er auf: journalistisches Arbeiten, journalistische Produktion, darin als Schwerpunkt Journalismus und Gesellschaft, ferner „Classic Journalism“, News Journalismus und Multimedia-Journalismus, jeweils auch mit Fokus auf digitale Techniken und Konzepte. Für den Einsatz in seiner Lehre dürfte das Lehrbuch sehr nützlich sein. Dem Fach generell bringt es wenig Neues, wenngleich Brinkmann Vieles, was beispielsweise Klaus Meier und Stephan RussMohl in mehreren Auflagen ihrer Lehrbücher aufbereiteten, durch eigene Überlegungen und Beispiele ergänzt. Zentral für ihn ist die Anwendung.

Sein Buch hat sieben Teile. Auf eine Einleitung, die Journalismus zwischen Handwerk und Haltung verortet, folgt ein einführendes Kapitel, in dem der Autor eine

Prof. Dr. Marlis Prinzing $(\bowtie)$

Hochschule Macromedia, Brüderstraße 17, 50667 Köln, Deutschland

E-Mail: m.prinzing@macromedia.de 
Definition von Journalismus vorschlägt, Bezüge zu Öffentlichkeit, Gesellschaft und demokratischem System aufzeigt und Journalismus gegen Werbung und PR abgrenzt, sowie eine allerdings nur sehr kurze theoretische Fundierung vornimmt. Im dritten Kapitel beschreibt Brinkmann politische, rechtliche, ethische und ökonomische Rahmenbedingungen, im vierten widmet er sich vor allem Berichterstattungskonzepten, Qualitätsfragen und der Redaktionsorganisation.

Daran schließt das mit 114 Seiten umfangreichste Kapitel in dem insgesamt 276 Seiten starken Lehrbuch an. In sechs Unterabschnitten beschreibt der Autor entlang konkreter Beispiele journalistische Arbeitsroutinen: von der Themenfindung über die Recherchen und die Wirklichkeitspräsentation in geeigneten Darstellungsformen bis hin zu Handreichungen für journalistisches Schreiben und Erzählen und der kontinuierlichen Vergewisserung der Faktizität insbesondere auch digitaler Informationen. Herzstück ist das Unterkapitel 5.3 mit den Darstellungsformen. Es hat 60 Seiten und ist zwar durch Zwischenüberschriften etwas strukturiert, aber hier würde eine weitere Nummerierung als Orientierung helfen. Die Genres werden Schritt für Schritt über Themenbeispiele beschrieben - die Form „Bericht“ anhand des Themas Clankriminalität, die Glosse an einem Aspekt aus der Corona-Thematik, die Reportage am Beispiel eines verarmten Münchner Rentners, das Feature an Food-Start-ups. Begründet mit dem knappen Platz, wurde der Fokus auf Print- und Onlineberichterstattung gelegt.

Lobenswert ist, dass die zentrale Rolle der Faktenüberprüfung gewürdigt wird, indem ihr ein eigenes Kapitel (5.6) zugedacht ist. Gerade deshalb wäre gut, die Schlüsselpunkte noch klarer und übersichtlich hervorzuheben sowie dem Teilaspekt der Redigier-Praxis zusätzlichen Raum zu geben, um ihrer Bedeutung Rechnung zu tragen. Das lässt sich auch auf das Redigat des Lehrbuch-Manuskripts beziehen: Auf S. 30 wird der Empfänger eines Medienbeitrags beschrieben als einer, der diesen „rezeptiert“ (statt rezipiert); der Hinweis, dass wir durch digitale Kommunikation Sender und Empfänger zugleich sein können, wäre idealerweise um den von Axel Bruns eingeführten Begriff des „Producers“ (oder „Produtzers“) ergänzt worden. Beim Bezug auf führende Denker der Frankfurter Schule (S. 49) ist neben Adorno und Horkheimer statt Marcuse von „Mabuse“ die Rede. Und im - ausführlichen Literaturverzeichnis heißt Rüdiger Funiok leider „Funiork“ (S. 258).

Das fünf als „Trends“ bezeichnete Entwicklungen beschreibende sechste Kapitel stellt Herausforderungen sowie Reaktionsweisen einander gegenüber: Techniken zum Debunken von Desinformation, Scrollytelling als digitale Erzählform, durch Big Data an Varianz gewinnender Datenjournalismus, durch mobile Geräte wie Smartphones mögliches Mobile Reporting sowie Social Media Praktiken als Berichterstattungshilfen oder Ausspielformen. Etwas unglücklich finde ich hierfür den Begriff „Social Journalism“, weil er leicht zu verwechseln ist mit sozialen Funktionen von Journalismus, die sich in Konzepten wie dem Konstruktiven Journalismus ausdrücken.

Brinkmann visualisiert seine Ausführungen in 60 Abbildungen und 18 Tabellen, für die er sich auf Darstellungen aus der angewandten Journalismusforschung stützt und sie aus seiner Perspektive heraus akzentuiert. Jedem Kapitel ist ein Abstract vorangestellt, abgesetzt sind Definitionen und vertiefende Erklärungen. Die Kapitel 
schließen - für Lehrbücher klassisch - mit Diskussionsfragen, die den Wissensstand prüfen, sowie mit Literaturtipps.

Das Lehrbuch behandelt Digitalisierung, Verantwortung und Haltung als Querschnittsaufgaben. Big Data und global agierende Betreiber von Social Media-Plattformen bewirken, dass sich Grenzen auflösen; Klimakrise, Pandemie und weitere komplexe Themen verunsichern viele Menschen. Journalismus ist Beobachterinstanz der sich verändernden Gesellschaft und selbst im Wandel. An entsprechende Befunde zur Medieninnovationsforschung knüpft das Schlusskapitel an und an den Kernauftrag von Journalismus. Er hat Bestand - und erfreulicherweise ebenso das Interesse der jungen Generation an diesem Beruf.

Funding Open Access funding enabled and organized by Projekt DEAL.

Open Access Dieser Artikel wird unter der Creative Commons Namensnennung 4.0 International Lizenz veröffentlicht, welche die Nutzung, Vervielfältigung, Bearbeitung, Verbreitung und Wiedergabe in jeglichem Medium und Format erlaubt, sofern Sie den/die ursprünglichen Autor(en) und die Quelle ordnungsgemäß nennen, einen Link zur Creative Commons Lizenz beifügen und angeben, ob Änderungen vorgenommen wurden.

Die in diesem Artikel enthaltenen Bilder und sonstiges Drittmaterial unterliegen ebenfalls der genannten Creative Commons Lizenz, sofern sich aus der Abbildungslegende nichts anderes ergibt. Sofern das betreffende Material nicht unter der genannten Creative Commons Lizenz steht und die betreffende Handlung nicht nach gesetzlichen Vorschriften erlaubt ist, ist für die oben aufgeführten Weiterverwendungen des Materials die Einwilligung des jeweiligen Rechteinhabers einzuholen.

Weitere Details zur Lizenz entnehmen Sie bitte der Lizenzinformation auf http://creativecommons.org/ licenses/by/4.0/deed.de.

Prof. Dr. Marlis Prinzing ist Professorin für Journalistik an der Macromedia Hochschule in Köln. 